\title{
Awareness of Pain Assessment for Elderly Patients with Dementia among Geriatric Care Hospital Nurses
}

\author{
Hyesun Park ${ }^{1}$, Sunyoung Lim$^{2}$ \\ ${ }^{1}$ Ph.D, Dept. of Red Cross College of Nursing, Chung-Ang University, Republic of Korea, \\ april1007@naver.com \\ ${ }^{2}$ Assistant Professor, Dept. of Nursing, Baekseok Culture University, Republic of Korea, \\ isokety@bscu.ac.kr \\ Corresponding author: Sunyoung Lim
}

\begin{abstract}
The purpose of this study was to examine the experiences of nurses as case managers in the pain assessment of elderly patients with dementia in geriatric care hospitals by conducting a qualitative case study. Data were collected through in-depth interviews with five nurses from two elderly care hospitals in Seoul and Gyeonggi Province. The collected data were qualitatively analyzed to examine experiences, strategies, and issues between the research cases. As a result of this study, five subcategories were derived: understanding of pain perception in dementia patients, difficulty in accurate self-report, assessment of pain behavioral indicators, identification of abnormal symptoms of pain through vital signs, and specific situation recognition. In addition, to verify the validity of the study, participant and peer reviews of the research results and interpretations were conducted. The results of the present study are intended to lay the basis for developing a pain assessment tool that can be used by nurses caring for elderly patients with dementia in geriatric care hospitals and pain management strategies. And, given the increasing importance of pain assessment and management through behavioral observation in the elderly with dementia in the clinical nursing practice for the elderly, a management plan for nurses must be prepared for pain assessment in elderly patients with dementia in geriatric care hospitals. The significance of this study is that it presents its results with supporting data.
\end{abstract}

Keywords: Geriatric Care Hospital, Dementia Elderly, Pain Assessment, Content Analysis Research

\section{Introduction}

In Korea, the number of elderly people with dementia has been increasing owing to the rapid aging of the population. The estimated number of patients with dementia aged $\geq 65$ years is 840,000 , accounting for $>10 \%$ of the total elderly population[1]. With the increasing number of patients with dementia, pain management in the elderly with dementia is not being performed properly because pain is a subjective experience and based on self-report. A previous study found that self-reported pain is difficult to assess, particularly in elderly patients with dementia in geriatric care hospitals, who have difficulty understanding the meaning conveyed with inconsistent words, repetitive actions, and expressions[2]. Even though the elderly with dementia have diseases that can cause pain, they cannot accurately recognize or express pain. Pain in the elderly with dementia impairs their daily life functions and causes psychological problems such as anxiety, depression, and sleep disorders, consequently reducing their quality of life[3]. 
The American Society of Geriatrics recommends considering patients' self-report of pain in pain management for elderly patients with dementia who have difficulty communicating but also recommends pain assessment through detailed behavioral observations[4]. Dementia in the elderly are divided into mild, moderate, and severe according to severity. As patients' verbal expression ability is also severely impaired, they have difficulty expressing pain and mainly express pain responses through non-verbal behaviors. Therefore, nurses must accurately assess pain in elderly patients with dementia in senior care hospitals, where most elderly patients have dementia[4-6].

Nurses who actually take care of and care for elderly patients with dementia in elderly care hospitals play a key role in pain assessment. In addition, nurses' objective and detailed observations for pain assessment can improve the self-integration and quality of life of elderly patients with dementia. Nurses taking care of elderly patients with dementia in geriatric care hospitals should check their patients' physical, cognitive, and mental functions. The cognitive function of the elderly with dementia is an essential factor for pain assessment[5][6]. Current domestic studies use self-assessment methods to assess pain, focusing on the community-dwelling elderly with mild dementia. However, self-evaluation is difficult among elderly patients with dementia in geriatric care hospitals because of cognitive impairment[4].

Moreover, the viewpoint and measurement subject are different from those of the tools for evaluating the effectiveness of pain interventions performed by nurses from the viewpoint of managing elderly patients with dementia[3]. The elderly with dementia experiences pain due to various diseases with old age, and affects their daily life function[3]. In addition, it is difficult to manage pain because it is not easy to determine whether the elderly with dementia have pain[2].Thus, pain assessment for the elderly with dementia in nursing homes for the elderly should be approached from the perspective of whether pain can be assessed and managed through detailed observations of nurses. Pain should be assessed and mediated from the perspective of nurses who manage the daily life of elderly patients with dementia[5][6].

When the elderly population increases and the number of elderly people with dementia increases accordingly owing to the increased life expectancy of the elderly in geriatric care hospitals, pain has become difficult to assess on the basis of self-report in these patients because of impaired cognitive function deterioration and verbal expression ability[1-3]. Therefore, the pain assessment of elderly care hospital nurses tending on the elderly with dementia is an important health management project. This study investigated the elderly pain assessment of nurses in elderly nursing hospitals caring for the elderly with dementia. It aims to contribute to providing basic data for the development of nursing interventions that can find ways to develop pain assessment tools and pain management for the elderly with dementia

\section{Methods}

\subsection{Research Design}

This is a descriptive qualitative study focused on analyzing interview responses using a content analysis method. Descriptive qualitative research is a research method that is aimed at expressing and comprehensively summarizing the event to be investigated in the language of participants without applying a specific philosophy or theoretical framework[7]. Accordingly, the main purpose of this study was not to analyze abstracted subjects derived from the data, rather, it intends to summarize and describe the data as they are. The interview was based on the research question "Tell me about assessing the pain of elderly patients with dementia while working as a nurse in a nursing home for the elderly?" 


\subsection{Research Subject}

The study participants were five nurses from geriatric care hospitals in Seoul and Gyeonggi-do, who were selected using an intentional sampling method. The research participants were selected among nurses with $>3$ years of experience in a nursing home for the elderly who met the theoretical qualification or competence to provide relevant information and data on the subject of this study [Table 1]. Data collection was conducted through individual in-depth interviews from July 15 to August 15, 2021. The location and time of the in-depth interview were decided in accordance with the wishes of the study participants. The interview duration was approximately 60 minutes per session. The interviews were conducted once or twice depending on the nurses' responses to the unstructured open-ended questions about pain assessment for the elderly with dementia in geriatric care hospitals and until the data were saturated.

[Table 1] Characteristics of the Respondents

\begin{tabular}{|c|c|c|c|c|}
\hline & Age(years) & Sex & $\begin{array}{c}\text { Total clinical experience } \\
\text { (years) }\end{array}$ & $\begin{array}{c}\text { Nursing hospital experience } \\
\text { (years) }\end{array}$ \\
\hline 1 & 36 & F & 9.2 & 3.6 \\
\hline 2 & 42 & F & 9 & 4.3 \\
\hline 3 & 44 & F & 8.5 & 3.8 \\
\hline 4 & 38 & F & 11 & 4 \\
\hline 5 & 39 & F & 10.6 & 3.5 \\
\hline
\end{tabular}

\subsection{Data Collection}

A questionnaire developed through a literature review was used to understand pain assessment for the elderly with dementia as perceived by nurses in the geriatric care hospital. The main question was "Tell me about assessing the pain of elderly patients with dementia while working as a nurse in a nursing home for the elderly." The interviews were conducted by two researchers who completed the qualitative research methodology and qualitative data analysis during their graduate studies and participated in qualitative conferences and workshops. Each interview session took an average of 60 minutes to complete. During the interview, non-verbal expressions such as speech, facial expressions, and gestures of the participants were observed and recorded in field notes. After obtaining consent to record the interview, a recorder was used. To secure the reliability of the data, the content of the recording was transcribed immediately after the interview and saved as a file.

\subsection{Data Analysis}

The traditional content analysis method was used for the data analysis. Content analysis is a systematic and objective analysis method for analyzing explicit or potential contents by classifying and evaluating contents to confirm the meaning and effect of major symbols or topics of data[8]. It is a dynamic analysis method that derives results inductively[9]. First, the researcher tried to understand the interview content as a whole by reading the transcripts and field notes repeatedly. The content was named and coded to better represent the pain situation of the elderly with dementia in the nursing homes for the elderly. Afterward, the process of categorizing and comparing the extracted codes according to meaning was repeated. Finally, the research question, "What is the perception of the elderly nursing 
hospital nurses about pain assessment for the elderly with dementia?," and subcategories were derived.

\subsection{Ethical Consideration}

The researcher explained to the participants the purpose and method of the study, the risks and benefits of participating in the interview, and the confidentiality measures. The participants were informed that they could withdraw their consent at any time during or after the interview and that they would not be put at any disadvantage if they wish to do so. They were informed that the collected data will be used only for research purposes and that the data of the participants will be kept strictly confidential. If they voluntarily consented to participate in the study, their written consent for study participation was obtained. During the interview, identifiable personal information was transcribed by replacing the symbols, and the recorded file was deleted after the transcription was completed.

\section{Results}

Five categories were derived from nurses' perceptions of pain assessment for the elderly with dementia in geriatric care hospitals [Table 2].

[Table 2] Essential Categories of Nurses' Pain Assessment for Elderly Patients with Dementia in Geriatric Care Hospitals

\begin{tabular}{c} 
Category \\
\hline Understanding the perception of pain in dementia patients \\
Difficulty in accurate self-reporting of pain \\
Assessment of behavioral indicators of pain \\
Identifying symptoms of pain based on vital signs \\
Awareness of the specific situation
\end{tabular}

\subsection{Understanding the Perception of Pain in Patients with Dementia}

We found that nurses in geriatric care hospitals should acknowledge and understand that pain assessment for elderly patients with dementia is different from that for the general elderly population, as expressed in the following statement:

"Most patients in the geriatric care hospital are elderly people with dementia, and we need to understand that there are many differences between how they express pain and how we perceive it compared with the general elderly population in the community. Ordinary elderly people do not have cognitive impairment, so they can evaluate pain on their own. Acknowledge and understand the differences between pain and suffering."

"Normal elderly people have little cognitive impairment, so they can evaluate their pain on their own, but the elderly in the geriatric care hospital are mostly elderly people with dementia, so there are many problem behaviors and many repetitive abnormal behaviors. Because it cannot accurately express the pain, it must be acknowledged and understood that it is different from the pain assessment of the general elderly."

"The pain assessment of the elderly with dementia at the Elderly Nursing Hospital is very different from that of the general elderly in the community. The general elderly in the community can evaluate their own pain for their pain because they have mild cognitive impairment. However, since the elderly in the geriatric care hospital are elderly people with dementia, the pain assessment must be evaluated by the nurse because the nurse must identify and evaluate the repetitive abnormal behaviors, that is, the parts shown in problem behaviors." 
"As a nurse in a nursing home for the elderly, I think that the pain situation of the elderly with dementia is different from that of general elderly patients. I think that elderly people with dementia in the elderly care hospital have many repetitive behaviors and inconsistent behavior due to cognitive impairment. Because the pain cannot be accurately expressed in words, the pain situation is different from that of the general elderly."

"As I work as a nurse in a nursing home for the elderly, the pain assessment of the elderly with dementia is different from the pain assessment that we generally assess for the elderly. And we need to acknowledge and understand the difference between the pain assessment and the general elderly patient. Ordinary elderly people have little cognitive impairment, so they can self-evaluate their pain, but elderly people with dementia at the geriatric care hospital cannot self-evaluate their pain."

\subsection{Difficulty in Accurate Self-reporting of Pain}

This category is a connecting context of the category of acknowledging and understanding that the perception of pain in the elderly with dementia is different from that of the general elderly population. We found that the nurses at the geriatric care hospitals recognized that other methods of pain assessments should be applied because the elderly with dementia did not accurately self-report their pain, as expressed in the following statement:

"The elderly with cognitive decline say that behavioral observation is necessary because they cannot report pain on their own and show pain through sobbing, grimacing, and gestures. Since it is difficult to self-report accurate pain, closely monitoring each and every action of the elderly will be helpful in assessing the pain of the elderly with dementia."

"Most of the elderly with dementia cannot self-report pain due to cognitive impairment, so we need to pay special attention to the non-verbal expression of the elderly with dementia. Whether they cry when there is pain, frown a lot, or point to a sore spot, you need to observe their actions one by one."

"Self-reporting of pain is not possible for elderly people with dementia in elderly care hospitals because their cognitive function varies from mild, moderate, and severe. In addition, since elderly people with dementia have more non-verbal expressions than verbal ones, it is important to carefully observe non-verbal expressions when they have pain. It is necessary to pay close attention to non-verbal and verbal expressions, such as crying because it hurts a lot, grimacing a lot, or moaning when pointing at a sore spot."

"As a nurse caring for the elderly with dementia at a geriatric care hospital, I acknowledge that the perception of pain in the elderly with dementia is different from that of the general elderly, and since the elderly with dementia do not accurately self-report pain, I believe that observable pain assessment should be done in a different way. Dementia elderly people with impaired function often express pain with gestures such as grimacing, sobbing, etc. This means that behavioral observation is necessary because such expressions express pain. Close observation of the patient will help assess pain in the elderly with dementia."

"The pain assessment of the elderly with dementia in the elderly care hospital that I observe is that the elderly with dementia cannot accurately self-report, so they need to assess pain that can be observed by other methods. Elderly people with dementia with cognitive decline cannot report pain on their own, so behavioral observations such as sobbing, grimacing, and gesturing are necessary."

\subsection{Assessment of Behavioral Indicators of Pain}

In this category, when the pain of patients with dementia was assessed, pain was recognized through behavioral indicators of pain, namely facial expressions, gestures, and postures, as expressed in the following statement: 
"When I observe the pain of the elderly with dementia, I recognize the pain through grimacing, moaning, worried faces, and sound diameters. Then, I check which part is painful again through physical assessment and apply pain management."

"When observing the pain of the elderly with dementia at the geriatric care hospital, I recognize the pain while reliving the ratio of focusing on verbal and non-verbal expressions according to the level of cognitive function. The elderly with dementia with mild cognitive impairment put more weight on verbal expression, the elderly with moderate dementia consider verbal and non-verbal expressions about half, and the elderly with severe dementia place more emphasis on non-verbal expression rather than verbal expression. Recognize pain with great emphasis on It is important to carefully observe the behavior of the elderly with dementia based on their physical circumstances. "

"I think it is necessary to assess the behavioral indicators of pain in the elderly with dementia. I think there is a difference in the degree of crying, grimacing, and screaming. And, according to the degree of cognitive function, pain is perceived with emphasis on both verbal and non-verbal expressions. Elderly people with mild cognitive impairment put more weight on verbal expression, elderly people with moderate dementia consider verbal and non-verbal expressions about half, and elderly people with severe dementia focus on non-verbal expression rather than verbal expression. Recognize pain with great weight. Both the degree of physical discomfort and the change in facial expression and moaning must be taken into account. "

"When caring for elderly people with dementia at a nursing home for the elderly, it is helpful to recognize pain through their facial expressions, gestures, and postures, that is, to understand well the behavioral indicators that complain of pain, especially in emotional situations. Pain can be assessed through grimacing, worried facial expressions, shouting, etc. This requires checking and understanding the physical and mental parts as well."

"I believe that when assessing the pain of dementia patients, it is necessary to properly identify the behavioral indicators that appear when complaining of pain. In other words, we take a closer look at the facial expressions of the elderly with dementia. Recognize pain through gestures and postures, such as whether you are grimacing, crying, or pointing and expressing pain."

"When I work at a nursing home for the elderly, when assessing the pain of dementia patients, I evaluate the pain while paying attention to the indicators that complain of pain, i.e., gestures, postures, and facial expressions, which are the behavioral indicators of the elderly with dementia. You will be able to assess different parts of your body gestures, closely examine the postures that are different from your usual postures, and see the differences in your facial expressions as well. Then you will see the overall physical condition as well as the psychological and psychological aspects."

\subsection{Identifying Symptoms of Pain based on Vital Signs}

In this category, when assessing pain in the elderly with dementia, symptoms of pain were recognized on the basis of vital signs, including blood pressure, heart rate, body temperature, and respiration, as expressed in the following statement:

"Most elderly with dementia have chronic diseases such as high blood pressure, diabetes, stroke, and arthritis, so it is necessary to distinguish whether the pain is due to an underlying disease and to accurately assess vital signs. It is important to know that grimacing or groaning has become more frequent, so when blood pressure is higher than usual, it should be considered as an indicator of pain in another part, and evaluation should be performed."

"When I am caring for a dementia patient in a nursing home for the elderly and assessing the pain, it is vital that I understand the pain along with the behavioral indicators. By assessing other parts of the body and measuring vital signs, it is possible to distinguish whether the elderly with dementia is a change in a chronic disease or a complaint of pain. It is necessary to accurately assess vital signs because it is 
possible to check whether the arthritis of the elderly with dementia has gotten worse."

"It is necessary to understand the vital signs of the elderly with dementia in the elderly care hospital. Most of the elderly with dementia have chronic diseases such as high blood pressure, stroke, diabetes, and arthritis, as well as cognitive impairment. Assessing vital signs is of paramount importance because blood pressure, pulse, body temperature, and respiration are largely related to pain."

"When assessing the pain of the elderly with dementia, I think that identifying abnormal signs through vital signs of blood pressure, pulse, body temperature, and respiration should be given the highest priority in pain assessment. Diseases that are important and that can come as other complications must also be considered."

"When I am assessing the pain of dementia patients in a nursing home for the elderly, I must identify abnormal symptoms of pain through vital signs. Knowing the changes in blood pressure, heart rate, body temperature, and respiration is identifying the pain assessment. Since elderly people with dementia have most of the chronic diseases, they will also be affected by the medications they take, and if there is even a slight change in the measurements of their usual vital signs, it is important to understand the cause. It is necessary to compare the vital signs of elderly people with dementia who have chronic diseases such as high blood pressure, diabetes, Parkinson's disease, stroke, and osteoporosis while taking conventional medications."

"I also measure vital signs and understand the condition of the emergency patient first, so vital signs are very important. Blood pressure, pulse rate per minute, respiration rate, and body temperature all play important roles, so pain assessment for the elderly with dementia is an important indicator for It is also necessary to consider the magnitude of the change in vital signs. I think that elderly people with dementia should be aware of both the level of blood pressure when they continue to take antihypertensive drugs and the change in blood pressure during pain assessment."

\subsection{Awareness of the Specific Situation}

The nurses in the geriatric care hospitals found that pain assessment in the elderly with dementia was helpful in understanding the circumstances where pain was more severe, as expressed in the following statement:

"I think it is important to understand what the current situation is when an elderly person with dementia complains of pain or shows pain through behavioral indicators. Whether the person complains of pain while resting or during movements such as during physical therapy, in order to manage pain only in a specific situation such as recognition of pain, it is necessary to look at the specific situation."

"As a nurse in a nursing home for the elderly, the first thing I need to do is to understand the current situation in order to assess the pain of the elderly with dementia. To say what the situation is is to know the specific situation. Identify the specific situations in which the elderly with dementia complains of pain when they are resting in bed, eating, walking in a wheelchair, participating in a program, or receiving physical therapy. You must be able to accurately assess the pain. And since cognitive impairment in the elderly with dementia has two aspects, which both hide the presence of pain and exacerbate the pain, I think that behavioral clues in specific situations should be well understood in the behavioral observation of the elderly with dementia."

"It is important to recognize the specific situation of the elderly with dementia in the elderly care hospital. Knowing in which situations the elderly with dementia exhibits more severe pain will help with pain assessment."

"I tend to observe the current situation and behavioral observations when elderly people with dementia at senior care hospitals complain of pain. Is the elderly with dementia complaining of pain even though they are walking in a wheelchair because they want to go for a walk? , It is necessary to recognize the specific situation because pain can be managed only by understanding the specific 
situation, whether the patient complains of pain when resting in bed or when receiving treatment."

"I think that when assessing the pain of the elderly with dementia, it is necessary to identify and recognize the specific situation. Nurses at the geriatric care hospital believe that it is helpful to assess the pain by understanding the circumstances in which the elderly with dementia complain of more severe pain. Pain can be managed only by understanding the specific situation, whether pain is expressed when lying in bed and resting, whether pain is expressed when performing certain movements or actions, whether pain is expressed when receiving physical therapy, occupational therapy, or a program. I think it is important, and in such a situation, it is necessary to evaluate the degree of verbal expression and changes in gestures, sounds, and behavioral postures together."

"I think it is important to figure out in which situation the pain is more severe in the pain assessment of the elderly with dementia. As usual, he complains of pain even when he is resting, and verbal and non-verbal expressions appear, and what movements... So, when receiving physical therapy, it is necessary to understand whether non-verbal expressions appear while complaining of pain."

\section{Discussion}

In this study, a content analysis was conducted using data from in-depth interviews to understand the pain assessment experiences and perceptions of nurses caring for elderly patients with dementia in geriatric care hospitals. From the categories of pain assessment recognition in the elderly with dementia by nurses in geriatric care hospitals, the following subcategories were derived: understanding the perception of pain in patients with dementia, difficulty in accurate self-reporting of pain, assessing behavioral indicators of pain, identifying symptoms of pain based on vital signs, and awareness of the specific situation.

First, the difficulty of understanding the perception of pain in patients with dementia and the difficulty of accurate self-reporting of pain are related to the same context. Pain is a highly subjective experience. In geriatric care hospitals, pain assessment for the elderly with dementia is based on cognitive skills such as language, memory, and judgment. Owing to disabilities, self-reporting is difficult and not useful for pain assessment; thus, pain must be assessed using the behavioral observation method[10]. In a previous study of the elderly with dementia, behavioral psychological symptoms increased as the duration of dementia spread was longer and pain was present[11]. In addition, dementia patients who can communicate and dementia patients who have difficulty communicating should be distinguished, and pain behavior observation tools for dementia patients should be continuously used[12]. Elderly patients with dementia in geriatric care hospitals are different from the general elderly population in the community in terms of their abilities to express and assess pain. Thus, nurses must understand these differences and perform pain assessment and management accordingly. In addition, pain management through pain assessment in the elderly with dementia is a very difficult process and is not properly performed. Pain is a subjective experience and should be based on self-report. Because people have difficulties in understanding the meaning conveyed through inconsistent words, repetitive actions, and gestures, it is more important to understand the pain perception of dementia patients and the difficulty of accurate self-report.

In addition, in the categories of assessing behavioral indicators of pain and identifying symptoms of pain based on vital signs, when nurses assess pain in elderly patients with dementia in a geriatric care hospital, they must identify the symptoms of pain on the basis of behavioral indicators and vital signs. During aging, various health problems such as cardiovascular, musculoskeletal, and peripheral nerve diseases; cancer; diseases requiring surgery; trauma; and chronic diseases occur frequently[13][14]. In addition, pain in patients with dementia experience impairs daily life functions and causes psychological problems such as depression, anxiety, and sleep disturbance, which in turn lowers quality of life[15].

Therefore, the pain-related behaviors of elderly patients with dementia, such as grimacing, moaning, 
worried face, and loud noises must be closely observed, and pain caused by underlying diseases must be distinguished from other pains in elderly patients in geriatric care hospitals, where most people with chronic diseases are elderly.

In addition, the "awareness of the specific situation" category is an important factor in pain assessment to determine the situation where the elderly with dementia experience more severe pain. To accurately assess pain only during movement such as when moving to the in observation, behavioral clues in specific situations should be well grasped[16][17].

Therefore, in this study, as the number of elderly people increases the same with the number of elderly people with dementia and the life expectancy at the elderly care hospital, it is difficult to self-report pain because the cognitive function of the elderly with dementia and verbal expression are impaired. Thus, it is meaningful to explore the perception of pain assessment for the elderly with dementia in elderly nursing hospital nurses to assess pain through inconsistent words, repetitive actions, and expressions.

\section{Conclusions}

This study was conducted to understand the nurses' perception of pain assessment for the elderly with dementia in geriatric care hospitals. In the results of this study, five subcategories were derived when nurses at elderly nursing hospitals assessed the pain of dementia patients.Understanding pain perception of dementia patients, difficulties in accurate self-reporting, assessment of behavioral indicators of pain, identification of abnormal signs of pain through vital signs, and recognition of specific situations were found to be important factors. This study has limitations in terms of generalizing its results, as it is a qualitative study conducted only among nurses from two geriatric care hospitals in Seoul and Gyeonggi Province. In the following study, continuous research is needed on nurses at many elderly nursing hospitals in various regions, and studies on pain perception based on verbal and non-verbal expressions of pain in elderly nursing hospitals are also needed.

However, given the increasing importance of pain assessment and management through behavioral observation in the elderly with dementia in the clinical nursing practice for the elderly, a management plan for nurses must be prepared for pain assessment in elderly patients with dementia in geriatric care hospitals. The significance of this study is that it presents its results with supporting data. The results of this study suggest the importance of developing nursing education and intervention programs for the evaluation of pain in elderly patients with dementia in geriatric care hospitals in the future and conducting studies to evaluate their effects.

\section{References}

[1] http://www.nid.or.kr/info/today_list.aspx, Aug 10(2021)

[2] J. Wang, P. Hsieh, C. Wang, Long-term Care Nurses' Communication Difficulties with People Living with Dementia in Taiwan, Asian Nursing Research, (2013), Vol.7, No.1, pp.99-103, DOI: https://doi.org/10.1016/j.anr.2013.06.001

[3] S. Jeoung, Effects of pain, activities of daily living, depression, and environment, on sleep quality among older adults with mild cognitive impairment dwelling in geriatric hospitals, Keimyung University, Master's thesis, pp.1-57, (2019)

[4] American Geriatrics Society Panel on the Pharmacological Management of Persistent Pain in Older Persons, Pharmacological management of persistent pain in older persons, Journal of the American Geriatrics Society, (2009), Vol.57, No.8. pp.1331-1346, DOI: https://doi.org/10.1111/j.1532-5415.2009.02376.x

[5] S. Lim, S. Chang, S. Kim, H. Kim, J. Choi, M. Park, Nurses' Management of Nursing Home Residents' Remaining Functional Ability: Concept Development, Journal of Korean Academy of Fundamentals of Nursing, (2014), Vol.21, No.1, pp.57-68, DOI: https://doi.org/10.7739/jkafn.2014.21.1.57 
[6] S. Lim, S. Chang, Ego-Integrity Management of Residents in Nursing Homes: A Concept Analysis based on the Method by Walker and Avant, Journal of Korean Gerontological Nursing, (2018), Vol.20, No.2, pp.97-108, DOI: https://doi.org/10.17079/jkgn.2018.20.2.97

[7] M. Sandelowski, Whatever happened to qualitative description?, Research in Nursing \& Health, (2000), Vol.23, No.4, pp.334-340, DOI: https://doi.org/10.1002/1098-240X(200008)23:4<334::AID-NUR9>3.0.CO;2-G

[8] K. Krippendorff, Content Analysis: an Introduction to Its Methodology, SAGE publication, p.196, (2018)

[9] H. Hsieh, S. Shannon, Three Approaches to Qualitative Content Analysis, Qualitative Health Research, (2005), Vol.15, No.9, pp.1277-1288, DOI: https://doi.org/10.1177/1049732305276687

[10] T. Hadjistavropoulos, K. D. Craig, A theoretical framework for understanding self-report and observational measures of pain: A communications model, Behaviour Research and Therapy, (2002), Vol.40, No.5, pp.551-570, DOI: https://doi.org/10.1016/S0005-7967(01)00072-9

[11] M. Lee, Relationship between pain and behavioral and psychological symptoms of dementia in nursing home residing elderly with dementia, Hanyang University, Master Thesis, pp.30-35, (2018)

[12] Y. Ryu, J. Park, Development and effect of evidence-based nursing practice guidelines for pain management in patients with dementia, Korean Journal of Adult Nursing, (2019), Vol.31, No.2, pp.176-189, DOI: https://doi.org/10.7475/kjan.2019.31.2.176

[13] A. Jablonski, M. Ersek, Nursing Home Staff Adherence to Evidence-Based Pain Management Practices, Journal of Gerontological Nursing, (2009), Vol.35, No.7, pp.28-34, DOI: https://doi.org/10.3928/00989134-20090428-03

[14] A. L. Horgas, A. F. Elliott, M. Marsiske, Pain Assessment in Persons with Dementia: Relationship Between SelfReport and Behavioral Observation, Journal of the American Geriatrics Society, (2009), Vol.57, No.1, pp.126-132, DOI: https://doi.org/10.1111/j.1532-5415.2008.02071.x

[15] K. Herr, Pain in the Older Adult: An Imperative Across All Health Care Settings, Pain Management Nursing, (2010), Vol.11, No.2, pp.S1-S10, DOI: https://doi.org/10.1016/j.pmn.2010.03.005

[16] S. Molony, M. Kobayashi, E. Holleran, M. Mezey, Assessing Pain as a FIFTH VITAL SIGN IN LONG-TERM CARE FACILITIES: Recommendations from the Field, Journal of Gerontological Nursing, (2005), Vol.31, No.3, pp.16-24, DOI: https://doi.org/10.3928/0098-9134-20050301-06

[17] A. L. Horgas, Assessing pain in persons with dementia, Dermatology Nursing, (2005), Vol.17, No.6, pp.472-473. 